\title{
ASSESSMENT FOR AN INCOMPLETE COMPARISON MATRIX AND IMPROVEMENT OF AN INCONSISTENT COMPARISON: COMPUTATIONAL EXPERIMENTS
}

\author{
Tsuneshi Obata ${ }^{\dagger}$, Shunsuke Shiraishi ${ }^{\ddagger}$, \\ Motomasa Daigo* and Nobuyuki Nakajima ${ }^{\ddagger}$ \\ $\dagger$ Faculty of Engineering, Oita University \\ Oita 870-1192, Japan \\ $\ddagger$ Faculty of Economics, Toyama University \\ Toyama 930-8555, Japan \\ *Faculty of Economics, Doshisha University \\ Kamigyou-ku, Kyoto 602-8580, Japan \\ obata@csis.oita-u.ac.jp shira@eco.toyama-u.ac.jp \\ mdaigo@mail.doshisha.ac.jp nakanobu@eco.toyama-u.ac.jp
}

\begin{abstract}
The characteristic polynomial of a positive reciprocal matrix has a noteworthy property. It is deeply related to the notion of consistency of a pairwise comparison matrix of AHP. Based on this property, we proposed an assessing method for an incomplete comparison matrix. In this paper, we report the results of computational experiments in order to investigate the validity and the performance of the method.
\end{abstract}

\section{Introduction}

In this paper we consider an $n \times n$ positive reciprocal matrix

$$
A=\left(\begin{array}{cccc}
1 & a_{12} & \cdots & a_{1 n} \\
1 / a_{12} & 1 & \cdots & a_{2 n} \\
\vdots & \vdots & \ddots & \vdots \\
1 / a_{1 n} & 1 / a_{2 n} & \cdots & 1
\end{array}\right)
$$

where $a_{i j}>0$ for all $i, j=1, \ldots, n$. In Analytic Hierarchy Process(AHP), positive reciprocal matrices appear as pairwise comparison matrices [Saa 80, Saa 94, Ton 86]. After completing the pairwise comparison matrix $A$, the priority weights $w=\left(w_{i}\right)$ are computed by Saaty's eigenvector method:

$$
A w=\lambda_{\max } w,
$$

where $\lambda_{\max }$ denotes the principal eigenvalue of $A$. By the eigenvector method, one also obtains the consistency index (C.I.) which is defined by:

$$
\text { C.I. }=\frac{\lambda_{\max }-n}{n-1}
$$

Decision makers are required to do pairwise comparisons so that C.I. would be as close to 0 as possible.

In [SOD 98], we examined the characteristic polynomial of $A$ :

$$
\begin{aligned}
P_{A}(\lambda) & =\operatorname{det}(\lambda E-A) \\
& =\lambda^{n}+c_{1} \lambda^{n-1}+\cdots+c_{n} .
\end{aligned}
$$


We showed that the coefficient $c_{3}$ of degree $n-3$ of $P_{A}$ plays a crucial role, that is, C.I. $=0$ if and only if $c_{3}=0$. Based on this fact, we proposed a method for assessing an incomplete pairwise comparison matrix (Section 2).

For assessing incomplete pairwise comparison matrices, Harker's method is used popularly [Har 87]. Our proposal is a heuristic method by which we expect to have a good C.I. value. That is to say, by putting $c_{3}$ close to 0 , we expect that C.I. is also close to 0 . In order to confirm our expectation, we make a computational experiment examining the relationship of $c_{3}$ and C.I. in Subsection 3.1.

Our method is useful when a decision maker encounters a seriously bad C.I. Make incomplete matrix by removing one element from the comparison matrix. Then assess the consistency of the incomplete matrix. If the consistency is improved remarkably, we recognize that the comparison corresponding to the removed element causes inconsistency. In Subsection 3.3, we make another experiment to examine validity of our method in this situation.

\section{Assessment Method for Incomplete Matrices}

In this section, we discuss a method to deal with incomplete pairwise matrices. First, we recall the following results from [SOD 98]. A positive reciprocal matrix $A$ is said to be consistent provided that $a_{i j} a_{j k}=a_{i k}$ for all $i, j, k=1, \ldots, n$.

Proposition 1 Let $n \geq 3$. If $A$ is a positive reciprocal $n \times n$ matrix, then

$$
c_{3}=\sum_{i<j<k}\left\{2-\left(\frac{a_{i j} a_{j k}}{a_{i k}}+\frac{a_{i k}}{a_{i j} a_{j k}}\right)\right\} .
$$

Theorem 1 Let $n \geq 3$ and $A$ be a positive reciprocal $n \times n$ matrix. Then we have:

1. $c_{3} \leq 0$

2. $A$ is consistent if and only if $c_{3}=0$.

The above theorem shows that one can judge the consistency of $A$ only from the coefficient $c_{3}$. Thus we find the following similarity between $c_{3}$ and the consistency index C.I. (see [Saa 80, Ton 86]). Indeed, a computational experiment shows a strong relation of their behaviors (see Subsection 3.1 below).

\section{observation}

$$
c_{3} \leq 0
$$

$c_{3}=0 \Leftrightarrow$ consistent

$$
\begin{aligned}
\text { C.I. } & \geq 0 \\
\text { C.I. }= & 0 \Leftrightarrow \text { consistent }
\end{aligned}
$$

Consider an incomplete pairwise comparison matrix whose $\left(i_{1}, j_{1}\right),\left(i_{2}, j_{2}\right), \ldots,\left(i_{m}, j_{m}\right)$ elements are missing. Denoting the value of the missing elements by $x=\left(x_{1}, x_{2}, \ldots, x_{m}\right)$ and the matrix by $A(x)$, we describe the incomplete matrix as follows:

$$
A(x)=\left(\begin{array}{cccccc}
1 & a_{12} & \cdots & x_{1} & \cdots & a_{1 n} \\
1 / a_{12} & 1 & \cdots & \cdots & \cdots & a_{2 n} \\
\vdots & \vdots & \ddots & & x_{2} & \vdots \\
1 / x_{1} & \vdots & & \ddots & & \vdots \\
\vdots & \vdots & 1 / x_{2} & & \ddots & \vdots \\
1 / a_{1 n} & 1 / a_{2 n} & \cdots & \cdots & \cdots & 1
\end{array}\right) .
$$


Since C.I. is required to be as small as possible, it is natural to seek the solution $x$ of the following minimization problem:

$$
\min _{x} \lambda_{\max }(x)
$$

where $\lambda_{\max }(x)$ denotes the principal eigenvalue of $A(x)$. However this problem is hard to solve exactly. Instead of solving (2), we proposed a heuristic method in [ODS 98]. Let $c_{3}(x)$ be the coefficient of $\lambda^{n-3}$ of the characteristic polynomial of $A(x)$. From the observation above, we expect that the consistency of $A(x)$ gets better as $c_{3}(x)$ gets closer to 0 . So we consider the following problem instead of (2):

$$
\max _{x} c_{3}(x)
$$

\section{[Proposed method]}

Step 1 Find the solution $x^{*}$ of the problem (3).

Step 2 Calculate the principal eigenvalue of $A\left(x^{*}\right)$ and its associate eigenvector.

Step 3 Normalize the eigenvector to a priority weight vector.

\section{Computational Experiments}

In this section we make some computational experiments in order to see the performance of our method.

\subsection{Relationship between $c_{3}$ and C.I.}

From Theorem 1, we expect that if $c_{3}$ is close to 0 , C.I. is also close to 0 . However this is not proven theoretically yet. Therefore we make the following computational experiment to see whether the expectation is appropriate or not.

\section{[Experiment 1]}

Step 1 Make a random comparison matrix $A$ of size $n$ (see Appendix).

Step 2 Calculate $c_{3}$ of $P_{A}$ and C.I. of $A$.

In Step 2, the value of $c_{3}$ is calculated by the representation formula (1) and C.I. by the power method, respectively. We repeated 1,000 times for each $n=4,5, \ldots, 15$. Fig. 1 shows the scatter plot of $c_{3}$ versus C.I. $(n=6)$. It looks like a comet with the long tail going toward the origin! In the cases other than $n=6$, the shapes are similar. From this shape, we are confident that the strong relation holds between their behaviors.

\subsection{Consistency of the method}

Our method aims to fill vacant elements as consistently as possible. On the other hand, Harker's method [Har 87] is designed to adjust the ideal situation. If the decision maker knows the real value of the priority weights $w=\left(w_{i}\right)$, then the comparison $a_{i j}$ should be $w_{i} / w_{j}$. By setting missing elements equal to $w_{i} / w_{j}$, he introduced a derived matrix and the associated eigenvector problem. So by his method, one also expects to assess incomplete matrices consistently. In [ODS 98, SOD 98], we made experiments in which we compared our method with Harker's method by calculating values of C.I. We don't mention the details here, but we can say that our method reveals better C.I. values than Harker's.

\subsection{Improvement of an inconsistent comparison}

When a pairwise comparison matrix contains seriously inconsistent comparisons, the priority weights calculated from such a wrong matrix are not reliable. Some researchers proposed methods for identifying the comparison which causes inconsistency and improving its value. Saaty [Saa 94, Chap. 3, Sec. 7] proposed the following method (we call it "Method-S"). 


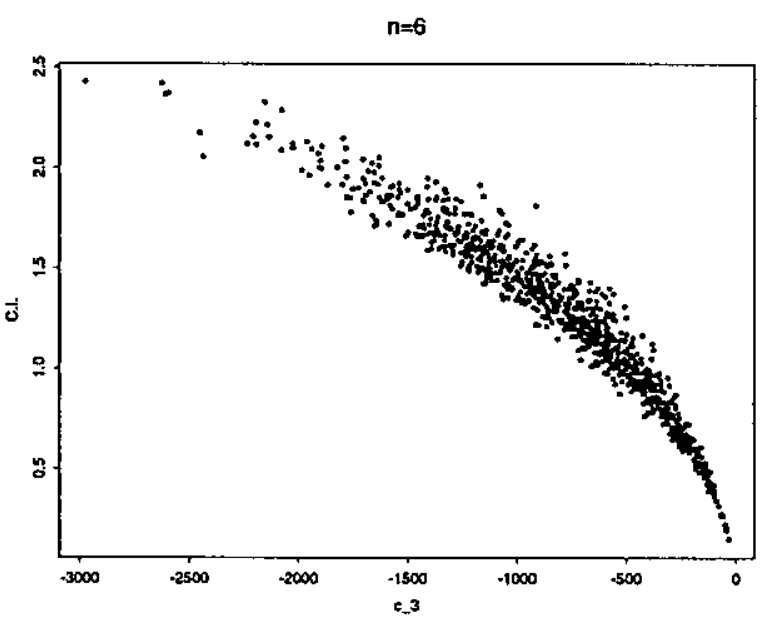

Figure 1: $c_{3}$ versus C.I. $(n=6)$

(i) Find the priority weight vector $w=\left(w_{i}\right)$ by the eigenvector method.

(ii) Compare each value $a_{i j}$ with the corresponding ratio of weights $w_{i} / w_{j}$.

(iii) Judge the most different element to be inconsistent, and replace $a_{i j}$ with $w_{i} / w_{j}$.

(iv) Estimate the priority weights from the modified matrix.

Once one obtains the assessing method for incomplete matrices, one naturally reaches the following idea (see [Nak 94]).

(i) Remove an element out of the comparison matrix.

(ii) Assess the consistency for the intentional incomplete matrix.

(iii) Estimate the weights when the consistency is improved most of all.

In assessing step (ii), one can use Harker's method or our method described in Section 2 (we call them "Method-H" and "Method-O", respectively).

In order to compare these methods, we make a matrix that includes an inconsistent comparison. In the following experiment, we intend to make a situation that the decision maker has almost completed good comparisons but she/he has come to fail only one comparison reciprocally reversed by chance (see Step 2 below).

\section{[Experiment 2]}

Step 1 Generate the original priority weight vector $w$ with degree $n$ randomly. Make an almost consistent comparison matrix $A$ with respect to $w$ (see Appendix).

Step 2 Exchange the $(1, n)$ element of the matrix $A$ for its reciprocal (if it equals to 1 then go back to Step 1).

Step 3 By using three methods, guess the element that causes inconsistency and estimate the weight vector, respectively;

Step 4 In order to investigate how good the estimation is made, calculate the square error from the original vector.

We repeated 1,000 times for each $n=4,5, \ldots, 15$. By this experiment, we examined the followings. (i) Can each of the methods identify $(1, n)$ element as a wrong comparison element? (ii) Can one estimate the appropriate weight vectors by each of the methods? 
Table 1 shows how many times each method failed to identify the inconsistent element. 'These

Table 1: \# of false

\begin{tabular}{c|cccccccccccc}
\hline$n$ & 4 & 5 & 6 & 7 & 8 & 9 & 10 & 11 & 12 & 13 & 14 & 15 \\
\hline $\mathrm{S}$ & 65 & 17 & 26 & 24 & 31 & 28 & 54 & 36 & 45 & 51 & 77 & 68 \\
$\mathrm{H}$ & 35 & 14 & 18 & 23 & 31 & 26 & 48 & 33 & 43 & 49 & 67 & 63 \\
$\mathrm{O}$ & 36 & 18 & 20 & 24 & 31 & 26 & 48 & 33 & 43 & 49 & 68 & 63 \\
\hline
\end{tabular}

mostly are the cases that the exchanged element equals to 2 or $1 / 2$.

Secondly, Table 2 shows the mean of square errors from the original vector. One may think that a method which reveals less error value can assess appropriate weights. The histograms in $n=6$

Table 2: mean of errors

\begin{tabular}{c|cccccc}
\hline$n$ & 4 & 5 & 6 & 7 & 8 & 9 \\
\hline $\mathrm{S}$ & .1184 & .0802 & .0566 & .0441 & .0357 & .0323 \\
$\mathrm{H}$ & .0555 & .0421 & .0368 & .0325 & .0286 & .0270 \\
$\mathrm{O}$ & .0555 & .0424 & .0370 & .0325 & .0286 & .0270 \\
\hline \hline$n$ & 10 & 11 & 12 & 13 & 14 & 15 \\
\hline $\mathrm{S}$ & .0286 & .0253 & .0241 & .0222 & .0208 & .0197 \\
$\mathrm{H}$ & .0250 & .0228 & .0219 & .0208 & .0197 & .0189 \\
$\mathrm{O}$ & .0250 & .0228 & .0219 & .0208 & .0197 & .0189 \\
\hline
\end{tabular}

are shown in Fig. 2 (Method-S, Method-H and Method-O from top to bottom).
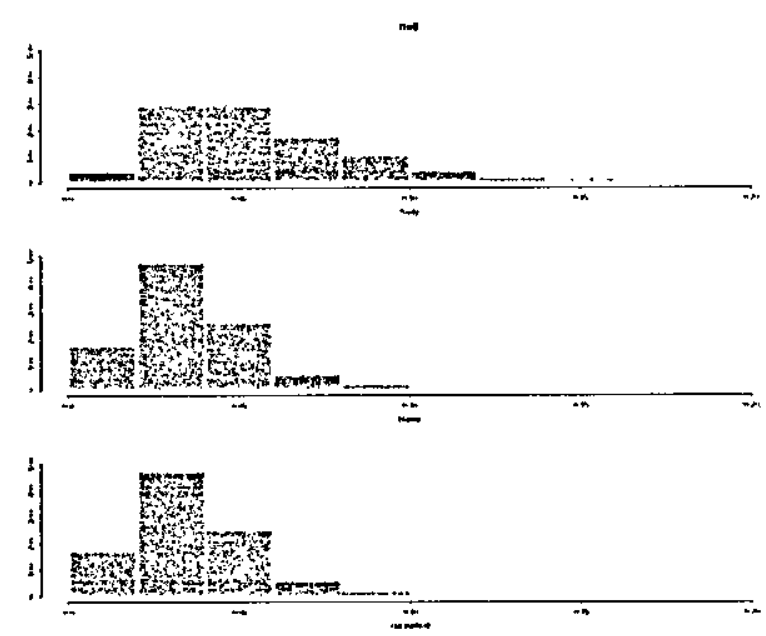

Figure 2: histograms of errors $(n=6)$

These results show that Method-H and Method-O are almost the same and they both reveal better performance than Method-S.

\section{Conclusion}

From the result of Experiment 1, we feel that there is a strong relationship between $c_{3}$ and C.I. Establishing theoretical relationship between $c_{3}$ and C.I. is left for future research. 
From the result of Experiment 2, on the occasion of improving consistency, our method is as useful as Harker's method while there is no striking domination. However, our method has the following advantage. It explicitly suggests the value of the missing element at the time just when one solves the problem (3). So we think that our method is more useful than Harker's in practical situations.

\section{Appendix}

Random comparison matrices of the size $n$ are made by the following procedure (see Experiment 1).

\section{[Random comparison matrix]}

Step 1 For $i<j$, generate $n(n-1) / 2$ numbers $a_{i j}$ from uniform random numbers in $1 / 9,1 / 8$, $\ldots, 1 / 2,1,2, \ldots, 8,9$.

Step 2 Set $a_{j i}=1 / a_{i j}$ to obtain a reciprocal matrix $A=\left(a_{i j}\right)$.

Almost consistent random comparison matrices are made by the following procedure (see Experiment 2).

\section{[Almost consistent matrix]}

Step 1 Generate $n$-vector from uniform random numbers in $(0,1)$. Normalize this vector and denote it the original weight vector $w=\left(w_{i}\right)$.

Step 2 Round the ratio $w_{i} / w_{j}$ to $1,2, \ldots, 9$ or its reciprocal and set $a_{i j}$ to the rounded value. Set $A=\left(a_{i j}\right)$.

\section{References}

[Har 87] Harker, P. T. (1987), "Alternative modes of questioning in the analytic hierarchy process," Mathl. Modelling, 9, 353-360.

[Nak 94] Nakajima, N. (1994), "Improvement of Inconsistency of Pairwise Comparison Matrices" (in Japanese), The Fudai Keizai Ronshu, 40, 229-244.

[ODS 98] Obata, T., Daigo, M. and Shiraishi, S. (1998), "A Computational Experiment on the Method for Estimating Priority Weights for Incomplete Comparison Matrices" (in Japanese), Reports of the FACUlty of ENGINEERING OITA UNIVERSITY, 37, 31-36.

[Saa 80] Saaty, T. L. (1980), The Analytic Hierarchy Process, McGraw-Hill.

[Saa 94] Saaty, T. L. (1994), The Analytic Hierarchy Process Series VI, RWS publication.

[SOD 98] Shiraishi, S., Obata, T. and Daigo, M. (1998), "Properties of a Positive Reciprocal Matrix and Their Application to AHP," Journal of the Operations Research Society of Japan, 41, 404-414.

[Ton 86] Tone, K. (1986), The Analytic Hierarchy Process: Decision Making (in Japanese), Japanese Science and Technology Press. 